\title{
Nuclear transparency in Monte Carlo neutrino event generators
}

\author{
Kajetan Niewczas ${ }^{1,2, *}$ and Jan T. Sobczyk ${ }^{1}$ \\ ${ }^{1}$ Institute of Theoretical Physics, University of Wroctaw, Plac Maxa Borna 9, 50-204 Wroctaw, Poland \\ ${ }^{2}$ Department of Physics and Astronomy, Ghent University, Proeftuinstraat 86, B-9000 Gent, Belgium
}

(Received 18 February 2019; revised manuscript received 17 May 2019; published 29 July 2019)

\begin{abstract}
The hadron cascade model is an essential part of Monte Carlo neutrino event generators that governs final-state interactions of knocked-out nucleons and produced pions. It is shown that such a model enriched with physically motivated modifications of nucleon-nucleon cross sections and incorporation of nuclear correlation effects is able to reproduce experimental nuclear transparency data. The uncertainty of nucleon final-state interaction effects is estimated and applied to recent neutrino-nucleus cross section measurements that include an outgoing proton in the experimental signal. Conclusions are drawn on a perspective of identification of events that originate from the two-body current mechanism.
\end{abstract}

DOI: 10.1103/PhysRevC.100.015505

\section{INTRODUCTION}

The description of the transport of hadrons in nuclear matter is a challenge encountered in many areas of fundamental research such as astrophysics, phenomenology of heavy-ion collisions, and a broad spectrum of nuclear physics applications. The first notable attempt of modeling this process was a Monte Carlo (MC) approach based on the ideas of Serber [1] and implemented by Metropolis et al. [2]. This concept of cascading hadrons was later followed by many others and the model developed significantly [3-6]. However, cascade models are based on theoretical assumptions which put limitations on their applicability $[1,7]$. This motivated researchers to develop two alternative solutions that go beyond this simplified picture and have been successfully used in, e.g., heavy-ion physics. The first one is based on BoltzmannUehling-Uhlenbeck (BUU) equations [8], formulated for the evolution of the one-body phase-space density under the influence of a mean field. The second one, known under the name of quantum molecular dynamics (QMD) [9], is formulated in terms of nucleon coordinates and momenta under the action of a many-body Hamiltonian. Both approaches are supplemented with a two-body collision term. A detail comparison of 15 independent implementations of BUU and QMD models shows surprisingly large differences in their predictions [10].

The transport problem is also of great importance for investigation of elementary projectiles scattering off atomic nuclei that involve final-state interactions (FSIs) of knockedout nucleons and produced mesons. It is particularly relevant

\footnotetext{
*kajetan.niewczas@uwr.edu.pl
}

Published by the American Physical Society under the terms of the Creative Commons Attribution 4.0 International license. Further distribution of this work must maintain attribution to the author $(\mathrm{s})$ and the published article's title, journal citation, and DOI. Funded by $S C O A P^{3}$. in neutrino physics, where further progress in reducing systematic errors in long- and short-baseline oscillation experiments [11] requires a more extensive use of measurements of final-state protons. On one hand, it is needed in calorimetric techniques to reconstruct neutrino energies. It is well established that more exclusive final-state measurements provide a better estimation of the neutrino energy $[12,13]$. Also, the investigation of final-state protons allows us to learn about the size of the multinucleon ejection contribution to the inclusive cross section, which is important even if neutrino energy is reconstructed based on the observation of the final-state muon only.

Analyses of oscillation experiments require reliable theoretical predictions for the complexity of nuclear responses to neutrino probes with broad energetic spectra. These predictions are obtained by using event generators, such as NEUT, GENIE, NuWro, and GiBUU [14]. The first three of them model FSIs using the intranuclear cascade model. An important test that the FSI model should pass is the ability to reproduce nuclear transparency data from electron scattering studies [15]. Nuclear transparency is defined as a probability that a knocked-out nucleon is not subject to reinteractions inside the residual nucleus. In the case of carbon targets, used in MINERvA [16] and T2K [17] experiments, typical transparency values are of the order of $65 \%$, and in the vast majority of events, knocked-out nucleons interact at most once.

The goal of this article is to present a procedure for checking if neutrino $\mathrm{MC}$ event generators reproduce nuclear transparency data. The discussion is done using the NuWro generator [18] but can easily be repeated with other neutrino $\mathrm{MC}$ event generators. Our main conclusion is that the NuWro nucleon cascade model, after enrichment of its physical content, describes nuclear transparency very well.

By comparing NuWro results with the electron transparency data we also estimated the uncertainty of the nucleon mean free path as calculated in the NuWro cascade model. Our conclusion is that if it is scaled up and down by $\sim 30 \%$ one 
gets an error bound that covers experimental uncertainties. With this estimate, we investigated how important is the impact of the FSI uncertainty for the recent $\mathrm{T} 2 \mathrm{~K}$ proton measurements. This defines a bound of experimental sensitivity in attempts to draw conclusions about the two-body current mechanism. Our conclusion is that nucleon FSI effects are controlled well enough to make the possibility of investigating the details of multinucleon ejection dynamics realistic.

The article is organized as follows. In Sec. II we describe how nuclear transparency is defined and measured in electron scattering experiments, following the procedures described in Refs. [19,20]. In Sec. III, the NuWro generator is presented with a focus on the description of the nucleon cascade model. Section IV contains the details of transparency computations in the NuWro generator. The last two sections, Secs. V and VI, contain a discussion of the results and our conclusions.

\section{NUCLEAR TRANSPARENCY}

Modeling of scattering processes on nuclear targets strongly relies on a description of nucleon propagation within nuclear medium. To estimate the magnitude of nucleon distortion, one can introduce a measure, called nuclear transparency, defined as the probability of a struck nucleon to escape the nucleus without significant reinteractions. Much attention has been brought to this subject following the hypothesis of color transparency (CT) [15]. Such a phenomenon should suppress the probability of in-medium nucleon-nucleon interaction at very high energies. CT has been extensively studied in many experiments, using quasifree $A\left(e, e^{\prime} p\right)$ scattering on various nuclei, so far without definite conclusions [15].

The general idea behind the measurement of the nuclear transparency in quasielastic $A\left(e, e^{\prime} p\right)$ reactions is to confront an experimental yield of knocked-out protons with a theoretical prediction that does not include the distortion due to FSIs. In these experiments, where an electron ejects a proton $p$ out of a nucleus $A$, using measured values of energy $\omega=E-E^{\prime}$ and momentum $\vec{q}=\vec{k}-\vec{k}^{\prime}$ transfers ( $E$ and $\vec{k}$ are the initial electron energy and momentum; primed values refer to the final electron), one defines the missing momentum and the missing energy as follows:

$$
\begin{aligned}
& \vec{p}_{m} \equiv \vec{p}_{p}-\vec{q}, \\
& E_{m} \equiv \omega-T_{p}-T_{A-1} .
\end{aligned}
$$

Here $T_{p}$ and $T_{A-1}=\left|\vec{p}_{m}\right|^{2} / 2 M_{A-1}$ are the kinetic energies of the knocked-out proton and the residual nucleus, respectively. The nuclear transparency, measured for a fixed fourmomentum transfer $Q^{2} \equiv|\vec{q}|^{2}-\omega^{2}$, is defined as

$$
T\left(Q^{2}\right)=\frac{\int_{V} d^{3} p_{m} d E_{m} Y_{\exp }\left(E_{m}, \vec{p}_{m}\right)}{\int_{V} d^{3} p_{m} d E_{m} Y_{\mathrm{PWIA}}\left(E_{m}, \vec{p}_{m}\right)},
$$

where $Y_{\exp }$ and $Y_{\text {PWIA }}$ are proton yields of the measurement and the theoretical calculation, respectively. The phase space $V$ is restricted to the quasielastic region by the conditions $E_{m} \lesssim 80 \mathrm{MeV}$ and $\left|\vec{p}_{m}\right| \lesssim 300 \mathrm{MeV}$, which ensure a suppression of inelastic processes. The theoretical prediction $Y_{\text {PWIA }}$ is calculated under a hypothesis of the plane-wave impulse approximation (PWIA), i.e., that the knocked-out nucleon does not undergo any reinteractions. One should be aware that the aforementioned definition suffers from a model dependency because it relies on the accuracy of theoretical PWIA computations.

Over the years, the following experiments have reported nuclear transparency measurements:

(i) D.F. Geesaman [21] and G. Garino et al. [22] at the Bates Linear Accelerator Center,

(ii) NE-18 at the Stanford Linear Accelerator Center (SLAC) [23,24],

(iii) E91-013 in Hall $\mathrm{C}$ at the Thomas Jefferson National Accelerator Facility (JLab) [19,20],

(iv) E94-139 in Hall $\mathrm{C}$ at the Thomas Jefferson National Accelerator Facility [25],

(v) E97-006 in Hall $\mathrm{C}$ at the Thomas Jefferson National Accelerator Facility [26].

The measurements were done in different kinematical setups, with outgoing protons' momenta in the range from $\sim 0.5$ to $\sim 5.5 \mathrm{GeV} / c$, and for various nuclear targets, with the most widely used being ${ }^{12} \mathrm{C}$ and ${ }^{56} \mathrm{Fe}$. The information about the kinematics of transparency measurements is summarized in Table I.

The PWIA models used by experimental groups describe the proton target in the independent particle shell models (IPSM). The IPSM-based calculations are known to overestimate single-particle strength in exclusive reactions [29]. This discrepancy is attributed to the shells that are not fully occupied due to nucleon-nucleon correlations that cannot be fully accounted for in mean-field approaches. NE-18 at SLAC was the first experiment that introduced the correlation factors $c_{A}$ in the definition of transparency to correct for the depletion of single-particle strength outside of the phase space $V$ :

$$
Y_{\mathrm{PWIA}}\left(E_{m}, \vec{p}_{m}\right)=c_{A} Y_{\mathrm{IPSM}}\left(E_{m}, \vec{p}_{m}\right) \text {, }
$$

with values $c_{A}=0.90$ and 0.82 for ${ }^{12} \mathrm{C}$ and ${ }^{56} \mathrm{Fe}$, respectively. They are larger than typically used spectroscopic factors, because they come up from the integration over a specific phase space $V$ [26]. In this article we compare our results to transparency results that they were published by experimental groups. Our treatment of correlation factors agrees with that from Ref. [30].

It has to be emphasized that the introduction of correlation factors is the subject of an ongoing debate. Some authors argue that because the experiments were conducted in the transverse kinematics, which is less sensitive to the highvalue tail of the nucleon momentum distribution, the use of correlation factors is not justified [31]. Theoretical arguments suggest that perhaps soft $Q^{2}$-dependent correlation factors would be more appropriate [32], but many recent articles on the nuclear transparency simply ignore them [15]. The CLAS Collaboration measured the nuclear transparency of protons from short-range correlated pairs [33] and arrived at the conclusion that the transparency ratios $\mathrm{Al} / \mathrm{C}, \mathrm{Fe} / \mathrm{C}$, and $\mathrm{Pb} / \mathrm{C}$ are consistent with the absence of the correlation factors in the definition Eq. (3). A similar conclusion is also supported by theoretical computations based on the Glauber theory [34-36] and the relativistic optical potential [34,35,37]. 
TABLE I. Kinematical setups of $A\left(e, e^{\prime} p\right)$ experiments that reported nuclear transparency measurements.

\begin{tabular}{|c|c|c|c|c|c|}
\hline Reference & $\begin{array}{l}\text { Beam } \\
\text { energy } \\
(\mathrm{MeV})\end{array}$ & $\begin{array}{c}\text { Central } \\
\text { electron } \\
\text { energy } \\
(\mathrm{MeV})\end{array}$ & $\begin{array}{c}\text { Central } \\
\text { electron } \\
\text { angle } \\
(\text { deg })\end{array}$ & $\begin{array}{c}\text { Central } \\
\text { proton } \\
\text { momentum } \\
(\mathrm{MeV} / c)\end{array}$ & $\begin{array}{l}\text { Central } \\
\text { proton } \\
\text { angle } \\
(\text { deg })\end{array}$ \\
\hline$[21,22]$ & 780 & 565 & 50.3 & 572.5 & $\begin{array}{l}50.1,58.2 \\
67.9,72.9\end{array}$ \\
\hline \multirow[t]{4}{*}[23,24]{} & 2015 & 1390 & 35.5 & 1200 & $\begin{array}{l}43.4,46.2, \\
49.0,51.8, \\
54.6\end{array}$ \\
\hline & 3188 & 1470 & 47.7 & 2450 & $\begin{array}{l}27.7,30.5, \\
33.3\end{array}$ \\
\hline & 4212 & 1470 & 53.4 & 3540 & $20.9,22.6$ \\
\hline & 5120 & 1470 & 56.6 & 4490 & 15.9, 16.7, \\
\hline \multirow[t]{6}{*}[19,20,27]{} & 2445 & 2075 & 20.5 & 882.8 & $\begin{array}{l}17.3 \\
35.4,39.4, \\
43.4,47.4, \\
51.4,55.4, \\
59.4,63.4, \\
67.4,71.4, \\
75.4\end{array}$ \\
\hline & 3245 & 2255 & 28.6 & 1661.7 & $\begin{array}{l}32.6,36.6, \\
40.6,44.6, \\
48.6,52.6\end{array}$ \\
\hline & 2445 & 1755 & 32.0 & 1343 & $\begin{array}{l}31.5,35.5, \\
39.5,43.5, \\
47.5,51.5, \\
55.5\end{array}$ \\
\hline & 3245 & 1400 & 50.0 & 2572.5 & $\begin{array}{l}25.5,28.0, \\
30.5\end{array}$ \\
\hline & 845 & 475 & 78.5 & 882.8 & $\begin{array}{l}27.8,31.8, \\
35.8,39.8, \\
43.8,47.8\end{array}$ \\
\hline & 1645 & 675 & 80.0 & 1661.7 & $22.8,26.8$ \\
\hline \multirow[t]{3}{*}[25,28]{} & 3059 & 1300 & 54.0 & 2520 & $\begin{array}{l}30.8,34.8 \\
19.8,22.3, \\
24.8,27.3, \\
29.8\end{array}$ \\
\hline & 4463 & 1200 & 64.6 & 4090 & 15.3 \\
\hline & 5560 & 1270 & 64.6 & 5150 & 12.8 \\
\hline \multirow[t]{5}{*}{ [26] } & 3298 & 2950 & 14.4 & 850 & 60.3 \\
\hline & 3298 & 2750 & 17.0 & 1000 & 56.2 \\
\hline & 3123 & 2500 & 22.2 & 1250 & 49.7 \\
\hline & 3298 & 2400 & 25.4 & 1500 & 44.6 \\
\hline & 3298 & 2280 & 29.0 & 1700 & 40.7 \\
\hline
\end{tabular}

\section{NuWro}

NuWro [18] is a neutrino Monte Carlo generator that has been developed at University of Wrocław since 2005. It covers neutrino energy range from $\sim 100 \mathrm{MeV}$ to $\sim 100 \mathrm{GeV}$. For neutrino-nucleon scattering NuWro uses three interaction "modes": CCQE (or elastic for neutral current reaction); RES, which covers a region of invariant hadronic mass $W<$ $1.6 \mathrm{GeV}$; and DIS (jargon in the neutrino MC community for shallow and deep inelastic scattering), in which the inelastic processes have $W>1.6 \mathrm{GeV}$. In the case of neutrino-nucleus scattering it is assumed that interactions occur on bound and moving nucleons (impulse approximation). A variety of options to describe such nucleons are available, including global and local Fermi gas (LFG) models up to the hole spectral function (SF) [38], with the lepton-affecting FSI effects included [39], and density- and momentum-dependent potentials [40]. The description of scattering off nuclear targets is completed with interactions mediated by meson exchange currents and with the coherent pion production.

For the purpose of this study the most important NuWro ingredient is the intranuclear cascade model described in Sec. III A.

\section{A. NuWro cascade model}

The model describes in-medium propagation of pions and nucleons. The scheme is taken from the seminal articles by Metropolis et al. [2,41] but relevant physics ingredients are new. The MC sampling is based on the standard formula that expresses the probability of a particle to propagate over a distance $\Delta x$ with no reinteraction:

$$
P(\Delta x)=\exp (-\Delta x / \lambda)
$$

where $\lambda=(\rho \sigma)^{-1}$ is the mean free path calculated locally, expressed in terms of the nuclear density $\rho$ and an effective interaction cross section $\sigma$. In actual computations we distinguish proton or neutron densities and proton-proton or proton-neutron cross sections. A step of $\Delta x=0.2 \mathrm{fm}$ was checked to be sufficient to grasp the structure of a nuclear density profile.

The performance of the NuWro pion cascade model was benchmarked on numerous neutrino-nucleus pion production cross-section measurements, showing in general a good agreement with the data (see, e.g., Ref. [42]). In this study, we focus on the nucleon cascade model.

The computations in this article were done with NuWro version 19.02 [43], which contains several improvements with respect to NuWro version 18.02. This version uses a custom fit to the experimental free nucleon-nucleon cross sections, both elastic and inelastic, that aimed to improve the agreement with the current PDG data set [14]. The fraction of single-pion production within inelastic interactions was adjusted to follow the fits of Ref. [44]. Moreover, the center-of-momentum (COM) frame angular distributions for the elastic scattering were updated using the parametrization of Ref. [45].

The in-medium modification of the elastic cross sections was modeled using the results of Pandharipande and Pieper's study [46], where the two main effects come from the Pauli blocking and the in-medium nucleon effective mass. The Pauli blocking is included on an event-by-event basis, a straightforward way in MC simulations. We checked that the NuWro cascade performance reproduces the results from Ref. [46] with sufficient accuracy. For the inelastic nucleon-nucleon scattering we adopt a phenomenological in-medium crosssection $\left(\sigma_{\mathrm{NN}}^{*}\right)$ parametrization [47]:

$$
\sigma_{\mathrm{NN}}^{*}=\left(1-\eta \frac{\rho}{\rho_{0}}\right) \sigma_{\mathrm{NN}}^{\text {free }},
$$


TABLE II. Table of cuts used by experimental groups and introduced in our simulations.

\begin{tabular}{|c|c|c|c|c|c|c|c|c|}
\hline$[21,22]$ & $\mathrm{C}, \mathrm{Fe}$ & 3.5 & 1.4 & 25 & 1.1 & 10.8 & 80 & - \\
\hline$[23,24]$ & $\mathrm{C}, \mathrm{Fe}$ & 5 & 0.9 & 5 & 0.9 & 4.6 & 100 & 250 \\
\hline$[19,20,27]$ & $\mathrm{C}, \mathrm{Fe}$ & 10 & 2.4 & 20 & 3.4 & 4.6 & 80 & 300 \\
\hline [26] & C & 9.6 & 2.4 & 15 & 3.4 & 7 & 80 & 300 \\
\hline
\end{tabular}

where $\eta=0.2$, and $\rho$ and $\rho_{0}$ are local and saturation nuclear densities, respectively.

Following the experiences of Refs. [30,36,46], we included effects coming from nucleon-nucleon short-range correlations. In general, the density that enters the mean free path in Eq. (5) is assumed to be the one of nuclear matter at point $\vec{r}_{2}$, as experienced by a propagating nucleon known to be in the position $\vec{r}_{1}$. It can be expressed in terms of one-body $\left(\rho_{\mathrm{A}}^{[1]}\right)$ and two-body $\left(\rho_{\mathrm{A}}^{[2]}\right)$ densities as

$$
\rho_{\mathrm{eff}}^{[1]}\left(\vec{r}_{2} \mid \vec{r}_{1}\right)=\frac{\rho_{\mathrm{A}}^{[2]}\left(\vec{r}_{1}, \vec{r}_{2}\right)}{\rho_{\mathrm{A}}^{[1]}\left(\vec{r}_{1}\right)},
$$

normalized to the number of remaining nucleons $\int d^{3} \vec{r}_{2} \rho_{\text {eff }}^{[1]}\left(\vec{r}_{2} \mid \vec{r}_{1}\right)=A-1$. We introduce correlation effects through the following substitution:

$$
\rho_{\mathrm{eff}, \text { IPSM }}^{[1]}\left(\vec{r}_{2} \mid \vec{r}_{1}\right)=\rho_{\mathrm{A}-1}^{[1]}\left(\vec{r}_{2}\right) \rightarrow \rho_{\mathrm{A}-1}^{[1]}\left(\vec{r}_{2}\right) g\left(\left|\vec{r}_{21}\right|\right) N\left(\left|\vec{r}_{2}\right|\right),
$$

where $g\left(\left|\vec{r}_{21}\right|\right)$ is the nucleus-dependent pair-distribution function [46] and $N\left(\left|\vec{r}_{2}\right|\right)$ is introduced to keep the global normalization condition.

For the choice of $g\left(\left|\vec{r}_{21}\right|\right)$, we rely on distributions of nucleon-nucleon distances obtained in ab initio computations for light nuclei, including carbon $[48,49]$. For heavier nuclei, including iron, we approximate $g\left(\left|\vec{r}_{21}\right|\right)$ by the $a b$ initiocalculated infinite nuclear matter distributions $g_{\text {inf }}\left(\rho_{\text {avg }},\left|\vec{r}_{21}\right|\right)$ of Ref. [46], evaluated at average nuclear density. In our computations we include effects coming from different shapes of $g\left(\left|\vec{r}_{21}\right|\right)$ for nucleon pairs of the distinct isospin configurations, and following the scheme summarized in Eq. (8) we define effective densities.

The discussion of the influence of the aforementioned NuWro cascade model modifications on the results of this article can be found in Sec. IV C.

\section{B. NuWro as a tool in transparency studies}

Using MC event generators one can define the "MC transparency" as a fraction of events with no nucleon reinteractions at all. However, experimentally one cannot distinguish these events from those with "soft" FSI. Because of that, to make a reliable comparison, we go through all the steps of the experimental procedures to extract the theoretical counterpart of the measured transparency.

NuWro keeps the information about particles before and after FSI. This is exactly what is needed in the computation of nuclear transparency. Particles after FSI correspond to those that are detected in experiments. Particles before FSI correspond to theoretical computations in PWIA.

NuWro does not yet have a complete electron scattering module; hence in this study we use neutral current (NC) $v_{e}$ interactions on bound proton targets. In doing so, we collect samples of NC events with exactly the same (electron mass is negligible) kinematics as in the transparency electron scattering experiments. In both electron and neutrino cases, the radial distribution of interaction points inside the nucleus is the same and given by the nucleus density profile.

The main challenge is to reproduce experimental situations with complete information on the kinematics and applied cuts. For every kinematical setup we ran a simulation with the neutrino beam energy equal to $E_{e}$. Then, the energy $E_{e^{\prime}}$ and the inplane angle $\theta_{e^{\prime}}$ for the outgoing electron or neutrino were fixed around the central value of the spectrometer. Analogically, the momentum $p_{p}$ and the in-plane angle $\theta_{p}$ for the knocked-out proton were fixed. As in all the experiments the electron and proton spectrometers were set in-plane, and the out-of-plane angles were fixed to the same value, $\phi_{e^{\prime}}=\phi_{p}$. The exclusive cross-section formula is symmetric with respect to the rotation of the system; hence only the relative out-of-plane angle between the electron and the proton plays a role, here set to $\phi_{e^{\prime} p}=0$. All of the variables, $E_{e^{\prime}}, \theta_{e^{\prime}}, p_{p}, \theta_{p}$, and $\phi_{e^{\prime} p}$, were fixed with the accuracy provided by the spectrometers' energy or angular acceptance, namely, $\Delta E_{e^{\prime}}, \Delta \theta_{e^{\prime}}, \Delta p_{p}, \Delta \theta_{p}$, and $\Delta \phi_{e^{\prime} p}=\Delta \phi_{e^{\prime}}+\Delta \phi_{p}$. On the top of those cuts, additional conditions were imposed using the information about $E_{m}$ and $\left|\vec{p}_{m}\right|$. The beam energies and central spectrometers values for every setup can be found in Table I, while the acceptances and the cuts on missing variables can be found in Table II.

To establish a proper framework for comparing nuclear transparency results with experimental results, we tested different ways of modeling the initial nuclear state in NuWro. The SF- and LFG-based simulations were compared with exclusive properties of the knocked-out protons that were reported by the E91-013 experiment at JLab. As can be seen in Fig. 1, the SF in NuWro is able to accurately reproduce a measured shape of the angular distributions of knocked-out protons. The angular dependence of transparency reproduces a general flat shape that can be seen in Fig. 2. of Ref. [19] with sufficient precision. However, the angular distribution of the measured yield of protons for the LFG-based simulations peaks too strongly around the central value, which leads to the overestimation of the proton transparency. Due 


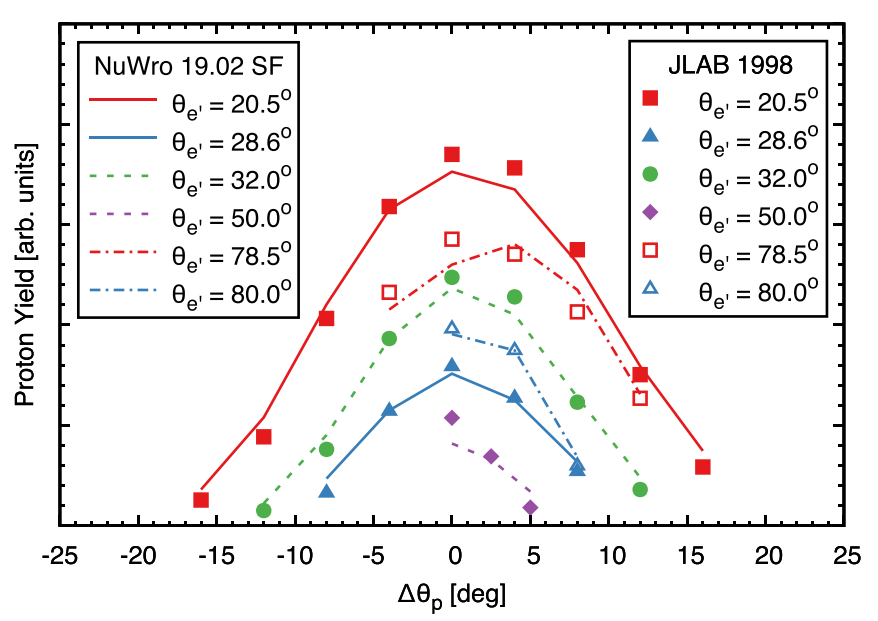

FIG. 1. Angular distributions of the proton yield as measured in the E91-013 experiment at JLab. Points represent data from Ref. [20] with the convention proposed in Ref. [27]. Lines are results computed with SF in NuWro. $\Delta \theta_{p}=0$ corresponds to the free proton target case. Both distributions are normalized to the same area.

to its simplicity, the LFG model fails to properly predict the exclusive kinematics, which is a prerequisite in reliable nuclear transparency studies.

We conclude that only NuWro simulations that use the $\mathrm{SF}$ as the model for the initial nuclear state can give reliable results in comparison with exclusive electron scattering experiments. Unfortunately, such a conclusion imposes a limitation on nuclear targets that can be simulated, because the hole spectral functions are available only for a limited number of nuclei making an estimation of the $A$ dependence of nuclear transparency impossible in NuWro. The only targets that can be compared with the transparency measurements are ${ }^{12} \mathrm{C}$ and ${ }^{56} \mathrm{Fe}$.

A similar study was done in the past using the Giessen BUU transport model [50,51]. The experimental data from three JLab and SLAC experiments [19,24,25] were analyzed using detailed information about the angular acceptance of spectrometers. Interesting ingredients of the BUU discussion are the following: the investigation of the impact of restricted angular acceptance on final results, a study of transparency dependence on atomic mass $T(A) \sim A^{\alpha}$, and an estimation of theoretical uncertainty due to not precisely known correlation factors $c_{A}[31]$.

The final BUU results are similar to the ones presented in this article as far as large proton momentum transparency saturation values are concerned. However, there is a visible difference at the lowest-momentum $\left(Q^{2}\right)$ experimental point: NuWro transparency continues to rise while the BUU transparency drops down.

\section{RESULTS}

In Fig. 2 the transparency results for carbon and iron are shown together with data points collected from several experiments. In experimental papers, transparency is discussed as a function of $Q^{2}$ but this variable can be translated into an average proton momentum. The transparency curve has a char-

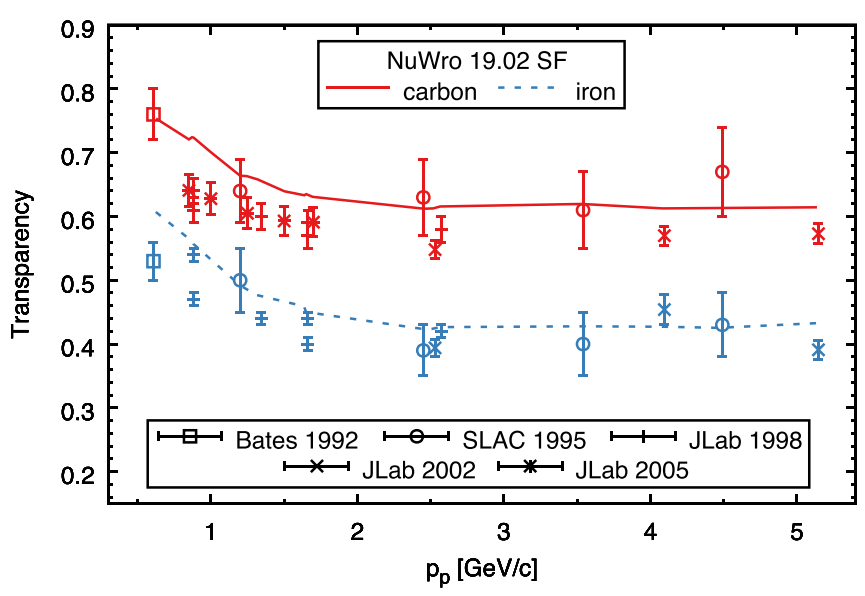

FIG. 2. Nuclear transparency as a function of proton momentum. Lines represent results obtained with NuWro 19.02 using the SF for carbon and iron targets. Experimental points come from experimental papers mentioned in the text.

acteristic shape reproduced in all theoretical computations: a saturation at larger values of proton momentum and a decline in the region of $\sim 1 \mathrm{GeV} / c$. Saturation can be explained by a roughly constant value of the total free nucleon-nucleon cross sections for larger values of the incident nucleon momentum. A region of transparency decline comes from a complicated interplay of various nuclear effects and is the most difficult to model.

NuWro simulations for carbon reproduce the transparency data quite well. For application in neutrino physics, the most important region is that of low nucleon momentum, starting from $\sim 500 \mathrm{MeV} / c$, which is a detection threshold in experiments like T2K and MINERvA. We can see that the value of the first available experimental point, from Ref. [22], is reproduced well but then the decline of NuWro transparency is not steep enough. Predictions from our model are slightly above the data in the saturation region. For the iron target, the same shape of the transparency curve can be seen. Small differences, including data overshooting at low momenta, can be attributed to nucleon-nucleon correlation effects being introduced in more approximate ways with respect to carbon (see the discussion in Sec. III A). In general, the agreement with the data points is satisfactory.

\section{A. Model uncertainties}

As discussed in the Introduction, nucleon FSI effects contribute to the background in all attempts to measure the multinucleon ejection contribution to the inclusive cross section. Thus, it is not enough to have good qualitative agreement with the transparency data; also it is important to estimate the uncertainty inherent in the nucleon FSI model. Our approach was to assess an uncertainty of the nucleon mean free path as calculated by NuWro. We tried to define a $1 \sigma$ error bound by demanding that $2 / 3$ of experimental points together with experimental errors be entirely inside the bound. To achieve that we multiplied the mean free paths calculated within NuWro by a constant overall scaling factor. The results are 


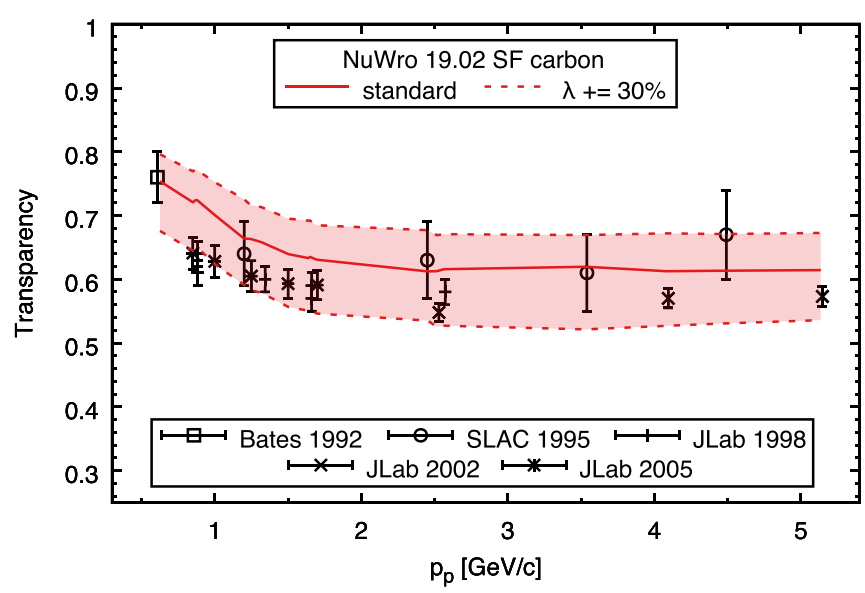

FIG. 3. Nuclear transparency as a function of proton momentum. Lines represent results obtained with NuWro 19.02 using the SF for the carbon target. Dashed lines are results computed after scaling mean free paths by $\pm 30 \%$. Experimental points come from the papers mentioned in the text.

shown in Fig. 3. The upper and lower dashed curves were obtained by scaling up and down the central mean free paths by $30 \%$. A discussion of possible sources of uncertainty in the NuWro FSI model is presented in Sec. IV C.

\section{B. Monte Carlo transparency}

In the MC approach, as mentioned in Sec. III B, a natural way of studying the nuclear transparency is to follow individual cascaded protons and check whether they interact at all. However as discussed earlier, such a definition might not catch particular aspects of the situation that are important from the experimental perspective and is expected to underestimate the final result. A refinement of the naive MC transparency definition is to take into account a finite angular acceptance of spectrometers and therefore allow protons to softly interact without a significant direction change, e.g., $\Delta \theta_{p}=5^{\circ}$. The value of $5^{\circ}$ approximately coincides with an angle that expands a solid angle in experimental acceptances (see Table II).

In Fig. 4, the results for carbon, using different transparency definitions, are shown. One can see that, while the "no interactions" definition is too strict, the softer definition " $\Delta \theta_{p}=5^{\circ}$ " works quite well, especially in the saturation region. However, it is unable to reproduce the first experimental point at $p_{p} \simeq 625 \mathrm{GeV} / c$. Knowing this behavior, the definition " $\Delta \theta_{p}=5^{\circ}$ " can be used for less exhausting cascade checks.

\section{Cascade model ingredients}

To understand sources of uncertainties in our model, we present the impact of its various ingredients on the predicted transparency. In Fig. 5, we show results obtained with the following:

(i) a bare cascade model with free nucleon-nucleon cross sections, projectile binding energy, and target nucleon Fermi motion effects;

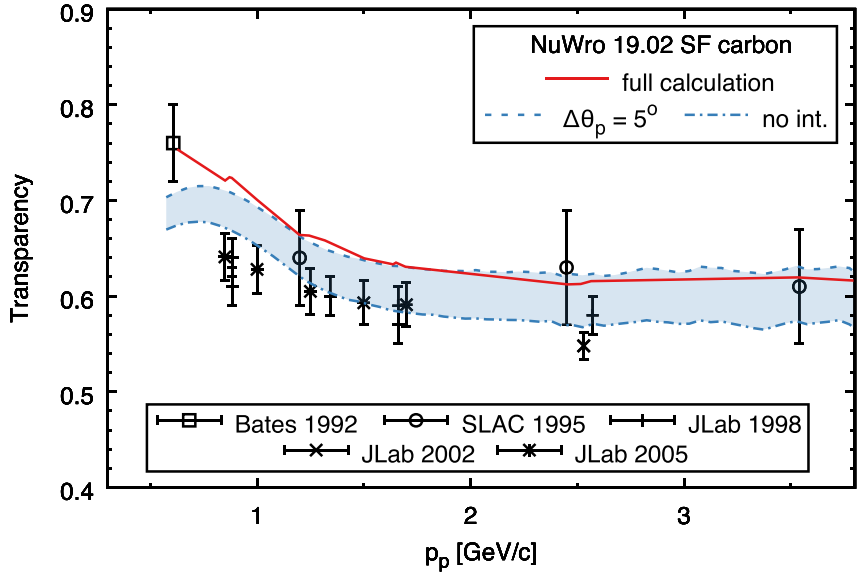

FIG. 4. Nuclear transparency, calculated with the full model and with the approximation discussed in Sec. IV B, as a function of proton momentum.

(ii) a model that on top of the bare model includes Pauli blocking (labeled "+ Pauli blocking");

(iii) a model that additionally includes in-medium nucleon-nucleon cross-section effects (labeled " + inmedium effects");

(iv) the full model that includes nucleon-nucleon correlation effects (labeled "+ correlations").

We can see that in the the region of proton momenta below $1 \mathrm{GeV} / c$ all the theoretical ingredients of the model are relevant, while for larger values of the momenta correlation effects play the most important role.

The basic observation about the bare model is that it underpredicts the experimentally measured transparency by a large amount. The proton momentum dependence of the corresponding curve reflects the momentum dependence of free proton-proton or proton-neutron cross sections. The effect of the Pauli blocking is significant for lower momenta and slowly disappears with the increasing proton momentum. Although it might not seem to be intuitive that the impact of

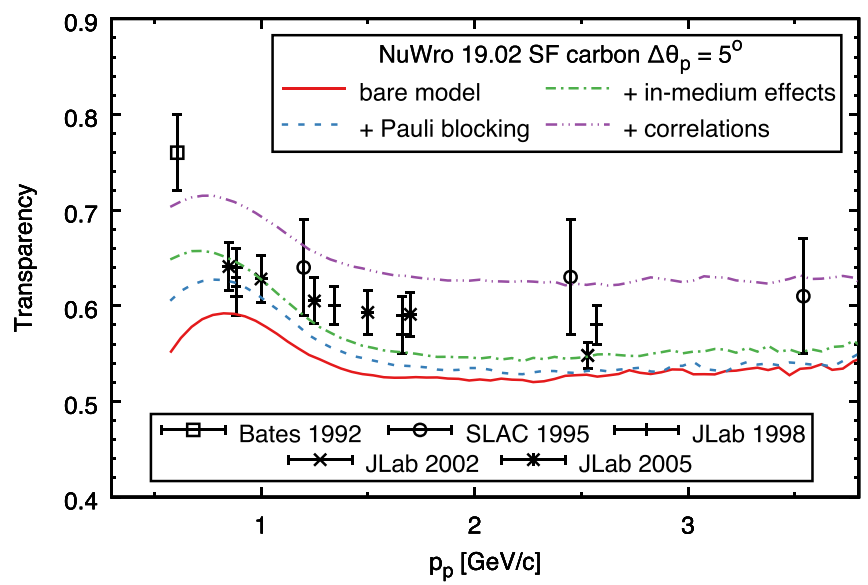

FIG. 5. Nuclear transparency as a function of proton momentum obtained with different ingredients of the theoretical model (see explanations in the text). 

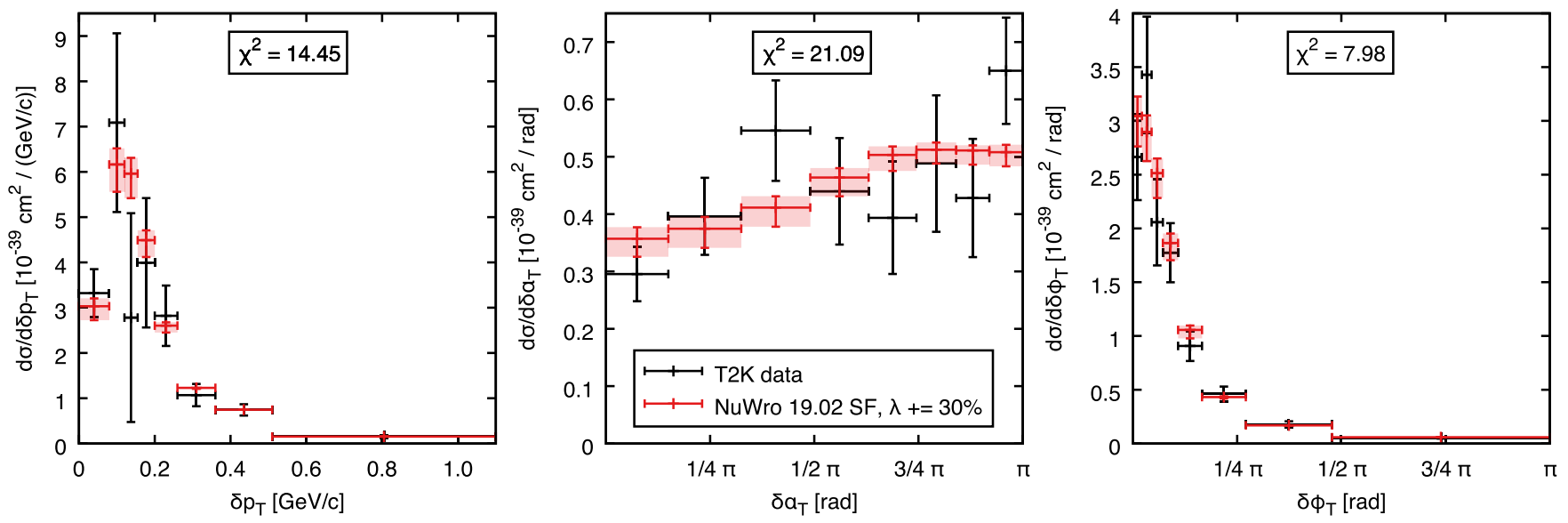

FIG. 6. Impact of FSI uncertainty in NuWro predictions for single transverse variables. Experimental points are taken from Ref. [71].

the Pauli blocking extends up to $p_{p} \simeq 2.5 \mathrm{GeV} / c$, for larger elastic scattering energies, the COM angular distributions get more and more forward or backward peaked, which leads to kinematics that are prone to be Pauli blocked. As emphasized in Sec. III A, the in-medium nucleon-nucleon cross-section modifications are modeled differently for elastic and inelastic interactions. This is reflected in nuclear transparency, where the modification of elastic cross sections has a stronger impact with lowering proton momentum, while the inelastic part has a constant behavior. The effect of the nuclear correlations strongly depends on average mean free paths in a given energy region. The free nucleon-nucleon cross section is higher in the saturation region, and therefore, the mean free paths are lower and the effect of correlations is more pronounced.

In general, all of the more sophisticated physical ingredients move the predicted transparency always in one direction, making it larger.

There is a significant difference of the behavior of transparency at the lowest values of proton momentum or $Q^{2}$ between the results presented here and the ones of Fig. 3 of Ref. [50]. A maximum at $Q^{2} \sim 1(\mathrm{GeV} / c)^{2}$, which is seen there, comes from the bare model maximum at $p_{p} \sim 0.8$ $\mathrm{GeV} / c$ (see Fig. 5). In our model, this structure mostly disappears when in-medium modification of the nucleon-nucleon cross sections are introduced.

\section{DISCUSSION}

The description of nuclear effects, and, in particular, the multinucleon ejection mechanism, is one of the crucial uncertainties in the neutrino oscillation analyses.

Although, many theoretical [52-60], experimental [61-65], and phenomenological [66-68] studies were done to increase the accuracy of the multinucleon ejection description, crude implementations in MC event generators limit the attempts to draw conclusions using more exclusive final states, e.g., with one muon and one proton in the final state. In the context of NuWro, as the remaining models either have satisfactory physical content (an exact SF implementation for the CCQE channel) or were successfully compared with data (for single-pion production, see Refs. [69,70]), one can attempt to investigate the seperation of multinucleon ejection events, assuming a proper control of the FSI modeling. In the following subsections, we present two applications of the aforementioned cascade model uncertainties on the MC predictions in the $\mathrm{CC} 0 \pi$ experimental channel.

\section{A. Application I: Single transverse variables}

As the first application, we discuss $\mathrm{T} 2 \mathrm{~K}$ measurements of single transverse variables [71]. These variables are defined in the following way:

$$
\begin{aligned}
& \delta p_{\mathrm{T}}=\left|\delta \vec{p}_{\mathrm{T}}\right|=\left|\left(\vec{p}_{p}\right)_{\mathrm{T}}+\vec{k}_{\mathrm{T}}^{\prime}\right|, \\
& \delta \alpha_{\mathrm{T}}=\arccos \frac{-\vec{k}_{\mathrm{T}}^{\prime} \cdot \delta \vec{p}_{\mathrm{T}}}{k_{\mathrm{T}}^{\prime} \delta p_{\mathrm{T}}}, \\
& \delta \phi_{\mathrm{T}}=\arccos \frac{\vec{k}_{\mathrm{T}}^{\prime} \cdot\left(\vec{p}_{p}\right)_{\mathrm{T}}}{k_{\mathrm{T}}^{\prime}\left(p_{p}\right)_{\mathrm{T}}} .
\end{aligned}
$$

Here $k^{\prime}$ and $p_{p}$ correspond to the outgoing lepton and proton, and index $\mathrm{T}$ denotes the transverse projection with respect to the beam direction. NuWro results are obtained with the SF model known to produce results better than those of the LFG model [71]. Due to many adjustments in the FSI model, the results obtained with NuWro 19.02 differ notably from those of older versions of NuWro. The most significant effect is an increase of normalization. This does not change much the values of $\chi^{2}$ for the SF-based results, but makes the $\chi^{2}$ values larger for the LFG-based ones.

In Fig. 6, we show how much uncertainty comes from possible NuWro FSI mismodeling. We see that applying a global decrease of the cascade mean free paths by $30 \%$ decreases the normalization of the results. We checked that this does not lead to a significant change of the calculated values of $\chi^{2}$. Making mean free paths $30 \%$ larger causes a slight increase of the value of $\chi^{2}$. A general conclusion is that for single transverse variables, the error coming from FSI strength seems to be well under control. 


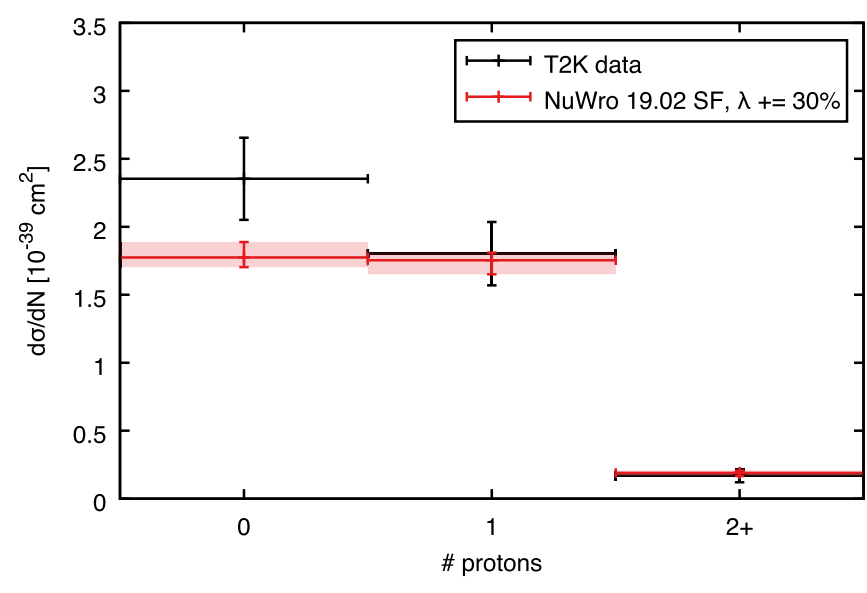

FIG. 7. Impact of FSI uncertainty in NuWro predictions for proton multiplicities. Experimental points are taken from Ref. [71].

\section{B. Application II: Proton multiplicities}

An observable that is potentially very sensitive to nucleon FSI effects is the distribution of the number of reconstructed protons. The dominant contribution to the experimental signal comes from CCQE events. Thanks to FSIs, there is a fraction of CCQE events with more than one proton; otherwise such events would be impossible. Another impact of FSIs is that due to rescattering some protons loose kinetic energy, dropping down below the detection threshold, which results in events with no detected protons. In general, the FSIs net effect is mostly a migration of events from $N=1$ to $N=0$.

In Fig. 7, we show a comparison of NuWro predictions with the T2K data from Ref. [71]. We see that the uncertainty coming from the unknown strength of FSIs is not large. Here, larger nucleon mean free paths result in increasing proton multiplicities. The data shape suggests that the FSI strength should be set at the biggest value acceptable by the nuclear transparency data. The impact of FSIs on the distribution is smaller than expected. This is because the experimental proton acceptance cuts eliminate most of the events with FSI.

\section{CONCLUSIONS}

NuWro 19.02 features an improved nucleon cascade model that, using proper comparison tools, is able to reproduce nuclear transparency data, in particular in the energy region that is crucial in the context of neutrino-nucleus scattering physics. The study presented in this article shows that a cascade model should be enriched with many additional effects, such as nucleon correlations, on top of a bare model with free nucleon-nucleon cross sections.

For the purpose of neutrino scattering physics, we estimated a $1 \sigma$ error on the nucleon mean free paths in NuWro 19.02 with a result of $30 \%$. This result was applied to recent T2K data that are potentially sensitive to nucleon FSI, giving an uncertainty that suggests that FSI modeling is under control and that there should be other sources of the data and MC disagreement that is still seen in NuWro results. There is a solid foundation for using these data sets in future research of multinucleon ejection contributions and especially a very uncertain hadronic part of its modeling [72].

All of the results obtained in this article can be easily reproduced with any other neutrino MC generator, such as NEUT or GENIE. The outcome of this work should allow one to further reduce systematic errors in the modeling of neutrino-nucleus scattering and, moreover, open a door for future analyses of more involved exclusive interaction channels.

\section{ACKNOWLEDGMENTS}

We thank T. Golan, C. Juszczak, O. Benhar, A. Lovato, W. Cosyn, N. Jachowicz, R. González Jiménez, A. Nikolakopoulos, J. Zalipska, and many of our T2K Collaborators for suggestions and discussions at various stages of this work. We thank J. Pasternak for reading the manuscript of this paper. The authors were supported by NCN Opus Grant No. 2016/21/B/ST2/01092 and also by the Polish Ministry of Science and Higher Education, Grant No. DIR/WK/2017/05. K.N. was partially supported by the Special Research Fund, Ghent University.
[1] R. Serber, Phys. Rev. 72, 1114 (1947).

[2] N. Metropolis, R. Bivins, M. Storm, A. Turkevich, J. M. Miller, and G. Friedlander, Phys. Rev. 110, 185 (1958).

[3] H. W. Bertini, Phys. Rev. 188, 1711 (1969).

[4] J. Cugnon, Phys. Rev. C 22, 1885 (1980).

[5] K. K. Gudima, S. G. Mashnik, and V. D. Toneev, Nucl. Phys. A 401, 329 (1983).

[6] T. T. Böhlen, F. Cerutti, M. P. W. Chin, A. Fassò, A. Ferrari, P. G. Ortega, A. Mairani, P. R. Sala, G. Smirnov, and V. Vlachoudis, Nucl. Data Sheets 120, 211 (2014).

[7] Y. Yariv et al., in Proceedings of the International Conference on Nuclear Data for Science and Technology, April 22-27, 2007, edited by O. Bersillon et al. (EDP Sciences, Les Ulis, 2008), p. 296.

[8] O. Buss, T. Gaitanos, K. Gallmeister, H. van Hees, M. Kaskulov, O. Lalakulich, A. B. Larionov, T. Leitner, J. Weil, and U. Mosel, Phys. Rep. 512, 1 (2012).

[9] J. Aichelin, Phys. Rep. 202, 233 (1991).
[10] Y.-X. Zhang et al., Phys. Rev. C 97, 034625 (2018).

[11] L. Alvarez-Ruso et al., Prog. Part. Nucl. Phys. 100, 1 (2018).

[12] U. Mosel, O. Lalakulich, and K. Gallmeister, Phys. Rev. Lett. 112, 151802 (2014).

[13] A. P. Furmanski and J. T. Sobczyk, Phys. Rev. C 95, 065501 (2017).

[14] H. Gallagher and Y. Hayato (Particle Data Group), Chin. Phys. C 40, 100001 (2016).

[15] D. Dutta, K. Hafidi, and M. Strikman, Prog. Part. Nucl. Phys. 69, 1 (2013).

[16] L. Aliaga et al. (MINERvA Collaboration), Nucl. Instrum. Methods Phys. Res., Sect. A 743, 130 (2014).

[17] K. Abe et al. (T2K Collaboration), Nucl. Instrum. Methods Phys. Res., Sect. A 659, 106 (2011).

[18] T. Golan, C. Juszczak, and J. T. Sobczyk, Phys. Rev. C 86, 015505 (2012).

[19] D. Abbott et al., Phys. Rev. Lett. 80, 5072 (1998).

[20] D. Dutta et al., Phys. Rev. C 68, 064603 (2003). 
[21] D. F. Geesaman et al., Phys. Rev. Lett. 63, 734 (1989).

[22] G. Garino et al., Phys. Rev. C 45, 780 (1992).

[23] N. C. R. Makins et al., Phys. Rev. Lett. 72, 1986 (1994).

[24] T. G. O'Neill et al., Phys. Lett. B 351, 87 (1995).

[25] K. Garrow et al., Phys. Rev. C 66, 044613 (2002).

[26] D. Rohe et al. (E97-006 Collaboration), Phys. Rev. C 72, 054602 (2005).

[27] D. Dutta, The $\left(e, e^{\prime} p\right)$ reaction mechanism in the quasielastic region, Ph.D. thesis, Northwestern University, 1999, Report No. UMI-99-32159.

[28] D. W. McKee, Nuclear transparency and single particle spectral functions from quasielastic $A\left(e, e^{\prime} p\right)$ reactions up to $Q^{2}=$ 8.1GeV ${ }^{2}$, Ph.D. thesis, New Mexico State University, 2003.

[29] J. M. Udias, P. Sarriguren, E. Moya de Guerra, E. Garrido, and J. A. Caballero, Phys. Rev. C 48, 2731 (1993).

[30] O. Benhar, Nucl. Phys. B, Proc. Suppl. 159, 168 (2006).

[31] L. Frankfurt, M. Strikman, and M. Zhalov, Phys. Lett. B 503, 73 (2001).

[32] L. Lapikas, G. van der Steenhoven, L. Frankfurt, M. Strikman, and M. Zhalov, Phys. Rev. C 61, 064325 (2000).

[33] O. Hen et al. (CLAS Collaboration), Phys. Lett. B 722, 63 (2013).

[34] P. Lava, M. C. Martinez, J. Ryckebusch, J. A. Caballero, and J. M. Udias, Phys. Lett. B 595, 177 (2004).

[35] M. C. Martinez, P. Lava, N. Jachowicz, J. Ryckebusch, K. Vantournhout, and J. M. Udias, Phys. Rev. C 73, 024607 (2006).

[36] W. Cosyn and J. Ryckebusch, Phys. Rev. C 87, 064608 (2013).

[37] J. J. Kelly, Phys. Rev. C 71, 064610 (2005).

[38] O. Benhar, A. Fabrocini, S. Fantoni, and I. Sick, Nucl. Phys. A 579, 493 (1994).

[39] A. M. Ankowski, O. Benhar, and M. Sakuda, Phys. Rev. D 91, 033005 (2015).

[40] C. Juszczak, J. A. Nowak, and J. T. Sobczyk, Eur. Phys. J. C 39, 195 (2005).

[41] N. Metropolis, R. Bivins, M. Storm, J. M. Miller, G. Friedlander, and A. Turkevich, Phys. Rev. 110, 204 (1958).

[42] C. L. McGivern et al. (MINERvA Collaboration), Phys. Rev. D 94, 052005 (2016).

[43] NuWro official repository, https://github.com/NuWro/nuwro.

[44] J. Bystricky, P. La France, F. Lehar, F. Perrot, T. Siemiarczuk, and P. Winternitz, J. Physique 48, 1901 (1987).

[45] J. Cugnon, J. Vandermeulen, and D. L'Hote, Nucl. Instrum. Methods Phys. Res., Sect. B 111, 215 (1996).

[46] V. R. Pandharipande and S. C. Pieper, Phys. Rev. C 45, 791 (1992).

[47] D. Klakow, G. Welke, and W. Bauer, Phys. Rev. C 48, 1982 (1993).

[48] Variational Monte Carlo calculations of two-nucleon densities, https://www.phy.anl.gov/theory/research/density2/.

[49] J. Carlson, S. Gandolfi, F. Pederiva, S. C. Pieper, R. Schiavilla, K. E. Schmidt, and R. B. Wiringa, Rev. Mod. Phys. 87, 1067 (2015).
[50] T. Leitner, O. Buss, and U. Mosel, Acta Phys. Pol. B 40, 2585 (2009).

[51] J. Lehr, In-medium-eigenschaftenvon nukleonen und nukleonresonanzen in einem semiklassischen transportmodell, $\mathrm{Ph} . \mathrm{D}$. thesis (in German), Giessen University, 2003.

[52] M. Martini, M. Ericson, G. Chanfray, and J. Marteau, Phys. Rev. C 81, 045502 (2010).

[53] M. Martini, M. Ericson, and G. Chanfray, Phys. Rev. C 84, 055502 (2011).

[54] J. Nieves, I. R. Simo, and M. J. Vicente Vacas, Phys. Rev. C 83, 045501 (2011).

[55] G. D. Megias, J. E. Amaro, M. B. Barbaro, J. A. Caballero, T. W. Donnelly, and I. R. Simo, Phys. Rev. D 94, 093004 (2016).

[56] T. Van Cuyck, N. Jachowicz, R. González-Jiménez, M. Martini, V. Pandey, J. Ryckebusch, and N. Van Dessel, Phys. Rev. C 94, 024611 (2016).

[57] T. Van Cuyck, N. Jachowicz, R. González-Jiménez, J. Ryckebusch, and N. Van Dessel, Phys. Rev. C 95, 054611 (2017).

[58] A. Lovato, S. Gandolfi, J. Carlson, E. Lusk, S. C. Pieper, and R. Schiavilla, Phys. Rev. C 97, 022502 (2018).

[59] N. Rocco, C. Barbieri, O. Benhar, A. De Pace, and A. Lovato, Phys. Rev. C 99, 025502 (2019).

[60] U. Mosel and K. Gallmeister, Phys. Rev. C 99, 035502 (2019)

[61] K. Abe et al. (T2K Collaboration), Phys. Rev. D 93, 112012 (2016).

[62] K. Abe et al. (T2K Collaboration), Phys. Rev. D 97, 012001 (2018).

[63] C. E. Patrick et al. (MINERvA Collaboration), Phys. Rev. D 97, 052002 (2018).

[64] R. Gran et al. (MINERvA Collaboration), Phys. Rev. Lett. 120, 221805 (2018).

[65] X.-G. Lu et al. (MINERvA Collaboration), Phys. Rev. Lett. 121, 022504 (2018).

[66] R. Gran, J. Nieves, F. Sanchez, and M. J. Vicente Vacas, Phys. Rev. D 88, 113007 (2013).

[67] K. Niewczas and J. T. Sobczyk, Phys. Rev. C 93, 035502 (2016)

[68] X. G. Lu, L. Pickering, S. Dolan, G. Barr, D. Coplowe, Y. Uchida, D. Wark, M. O. Wascko, A. Weber, and T. Yuan, Phys. Rev. C 94, 015503 (2016).

[69] R. González-Jiménez, K. Niewczas, and N. Jachowicz, Phys. Rev. D 97, 013004 (2018).

[70] A. Nikolakopoulos, R. González-Jiménez, K. Niewczas, J. Sobczyk, and N. Jachowicz, Phys. Rev. D 97, 093008 (2018).

[71] K. Abe et al. (T2K Collaboration), Phys. Rev. D 98, 032003 (2018).

[72] J. T. Sobczyk, Phys. Rev. C 86, 015504 (2012). 